\title{
Diagnostics for plasma control on DEMO: challenges of implementation
}

\author{
A.J.H. Donnée ${ }^{a, b}$, A.E. Costley ${ }^{c}$ and A.W. Morris ${ }^{c}$ \\ ${ }^{a}$ Dutch Institute For Fundamental Energy Research, Association EURATOM-FOM, \\ PO Box 1207, 3430 BE Nieuwegein, The Netherlands \\ ${ }^{\mathrm{b}}$ Eindhoven University of Technology, Dept. of Applied Physics, PO Box 513, \\ 5600 MB Eindhoven, The Netherlands \\ ${ }^{\mathrm{c}}$ EURATOM/CCFE Fusion Association, Culham Science Centre, Abingdon, \\ Oxfordshire OX14 3DB, UK
}

E-mail: donne@rijnhuizen.nl

\begin{abstract}
As a test fusion power plant, DEMO will have to demonstrate reliability and very long pulse/steady-state operation, which calls for unprecedented robustness and reliability of all diagnostic systems (also requiring adequate redundancy). But DEMO will have higher levels of neutron and gamma fluxes, and fluences, nuclear heating, and fluxes of particles than ITER, and probably reduced physical access. In particular, the neutron fluence will be about 15-50 times higher than in ITER. As a consequence, some diagnostics that will work in ITER are likely to be unfeasible in DEMO. It is important, therefore, to develop a new way of thinking with respect to that employed to date in which diagnostics are added after the machine has been basically designed: if certain diagnostics are deemed essential for the control of DEMO, they will have to be taken into account during the entire design phase.
\end{abstract}

\section{Introduction}

DEMO is the next-step magnetic fusion device that is intended to follow ITER. In practice, because the development timescales are long (many years), while the need to proceed with fusion development is considerable, it may partially overlap ITER. There are different views of what role this next step device should fulfill. ${ }^{1,2,3,4,5}$ In Europe, the predominant view is that DEMO should be the only step between ITER and a commercial power plant. Since ITER will not address all scientific and technological challenges that need to be overcome before a reactor can be built, there will still be developmental aspects to DEMO. In other countries engaged in fusion research, for example the United States, the line of thinking is that DEMO, as the last step before a commercial reactor, must have minimum developmental aspects: it must be a 'first of a kind reactor'. ${ }^{2}$ In this case there needs to be a step between ITER and DEMO. Most of the DEMO studies concentrate on the tokamak concept. However, studies of DEMO stellarators are also being conducted. ${ }^{5,6}$ In Europe a study has been undertaken ${ }^{1}$ to evaluate various possible designs for tokamak-based power plants that could be built after DEMO. Various designs are being studied, with major radius, $R$, varying from 6 to $9.5 \mathrm{~m}$ and with fusion power $P_{\text {fus }}$ from 2.5 to $5.0 \mathrm{GW}$. Figure 1 shows the plasma cross sections of the two most extreme designs along with that of ITER. 


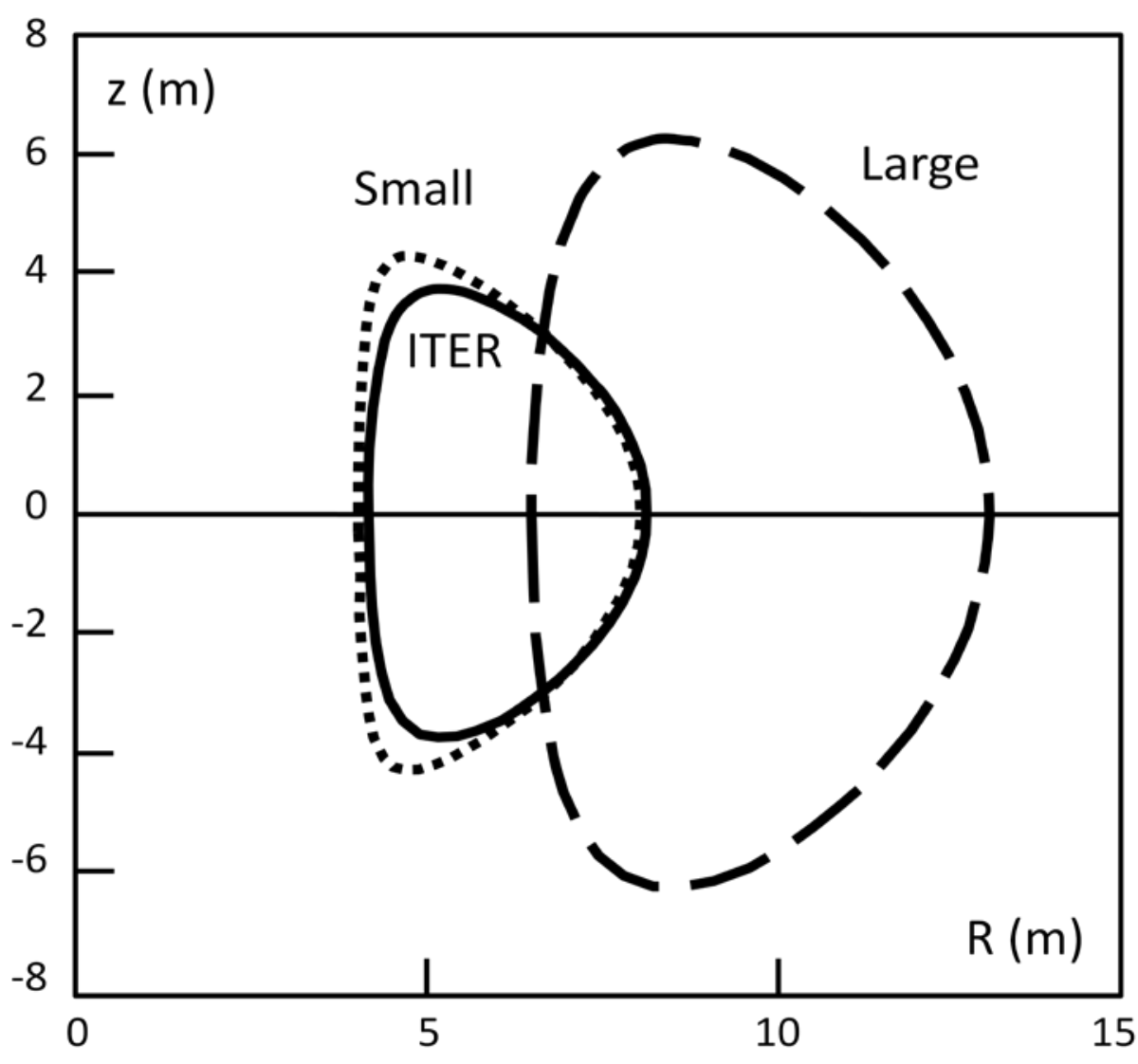

Figure 1. Illustration of the extremes of the plasmas in the various European power plant models. ${ }^{1}$ For comparison ITER is also shown. The axis labels denote major radius $(R)$ and height $(\mathrm{z})$. (Figure adapted from the figure in ref. [1]).

The European aim is to focus on just two DEMO options ${ }^{7}$ (note that the electrical power output from a fusion plant would be steady-state whether or not the tokamak is operated cyclically):

a. a "conservative baseline design" called an early DEMO concept deliverable in the short to medium term (e.g. construction to be started in 20 years from now), based on the expected performance of ITER with reasonable improvements in science and technology; i.e., a large, modest power density, long-pulse inductively supported plasma in a conventional plasma scenario. This version of DEMO is most likely to be close to the larger power plant design (dashed) in Fig 1.

b. an "optimistic design”, i.e, a DEMO concept based around more advanced physics and technology assumptions which are at the upper limit of what may be achieved in ITER phase 2, i.e. an advanced higher power density high CD steady state plasma scenario. It is clear that this can only be delivered on a longer term. This version of DEMO is most likely to be close to the smaller power plant design (dotted) in Fig 1.

It is possible to get an initial indication of the parameters that need to be controlled in the two European DEMO designs, by comparing the parameters that are to be included in the control loops of the ITER ELMy H-mode phase and the ITER steady-state phase (see Table 1, derived from a more extensive table published in ref. [8]). Assuming that the control needs for the two DEMO designs will be similar to those of the two ITER phases, one can see that there are sufficient generic similarities between the two to make a study of the diagnostic aspects worthwhile at this early stage. The smaller 
steady-state design will have to operate closer to operational boundaries and will on present understanding require control of additional parameters, such as q-profile, $T_{i}$ and $T_{e}$ profiles (especially gradients in these parameters) and Resistive Wall Modes.

Table 1. Indicative list of parameters that would ideally be measured in the two DEMO designs (derived from the diagnostic needs for the ITER ELMy H-mode and steady state phases [8]), as starting point for simplification

\begin{tabular}{ll}
\hline DEMO design/ITER phase & Plasma parameters to be measured for control and protection on \\
& ITER \\
\hline Long-pulse ELMy H-mode & Plasma shape and position, vertical speed, toroidal field, plasma current, \\
& loop voltage, line-averaged density, $\mathrm{T}_{\mathrm{i}}$ and $\mathrm{T}_{\mathrm{e}}$ in the plasma core, fusion \\
& power, radiated power, toroidal and poloidal rotation velocities, helium \\
& density profile, fuel ratio in plasma core, localization of $\mathrm{q}=1.5$ and $\mathrm{q}=2$ \\
& surfaces, locked modes, low $\mathrm{m} / \mathrm{n}$ MHD modes (in particular detection of \\
& NTMs), halo currents, runaway electrons, surface temperature of divertor \\
& plates and first wall, H/L mode indicator, ELM occurrence and type, \\
& divertor detachment, divertor ionisation front position \\
\hline Steady State & $\begin{array}{l}\text { All the same measurements as above in steady state, plus in addition: } \mathrm{q}- \\
\text { profile (in particular the location and value of } \mathrm{q}_{\text {min }} \text { ), high resolution } \\
\\
\text { measurements of the gradients in } \mathrm{T}_{\mathrm{i}} \text { and } \mathrm{T}_{\mathrm{e}}, \text { detection of RWMs. }\end{array}$ \\
\hline
\end{tabular}

The paper addresses specifically the challenges for diagnostics that will be applied in DEMO for machine protection and control purposes. In Section 2 the focus will be on the harsh environmental effects and also on the relativistic effects. Alpha-particle effects are dealt with in Section 3. Development of diagnostics for DEMO, including some possible suggestions for overcoming the implementation problems, are discussed in Section 4, followed by an overview of the design process in Section 5.

\section{Environmental effects}

DEMO will have higher levels of neutron and gamma fluxes, and fluences, nuclear heating, particle fluences (from charge exchange reactions) than ITER (see Table 2). In particular, the neutron fluence will be 15-50 times higher than in ITER, while the neutron flux is a few times higher. This implies that some techniques or diagnostic components that work in the ITER environment, ${ }^{8}$ are likely to be unfeasible in DEMO. Figure 2 presents an overview of the most important environmental effects on the diagnostic components that are located at close proximity to the ITER plasma. ${ }^{9}$ Electrical components (e.g. mineral insulated cables, magnetic coils, electric wiring) can suffer from a large range of environmental effects including radiation induced conductivity (RIC), radiation induced electric degradation (RIED), radiation induced electromotive force (RIEMF), thermal induced electromotive force (TIEMF), radiation induced thermo-electric sensitivity (RITES). Further, electrical circuits can suffer from contact degradation and transmutation (e.g. gold meanders on bolometers transmute to mercury, which prevents their proper operation). Considerable and successful R\&D would be needed to make it possible to use them in DEMO in a similar way as in ITER. Refractive components (lenses, windows, fibers) suffer from radiation induced absorption (RIA; which effectively causes the components to become opaque) and radiation induced emission (RIE or radioluminescence). These components can therefore not be used at close proximity to the plasma. Instead of refractive components, mirrors must be used in a wide wavelength range. But mirrors suffer from erosion and deposition by particle bombardments from the plasma. The particle flux in DEMO is 
expected to be similar to that in ITER but the particle fluence will be many times higher (possibly two orders of magnitude). As a consequence, optical systems with large-aperture mirrors may be unfeasible in DEMO. Further, the neutron flux and nuclear heating may lead to swelling, changing the shape of the mirrors, and hence, affect their imaging qualities. Small apertures and mirrors recessed far into the shielding, and use of exchangeable optical fibres, could make optical diagnosis possible, but with very limited views of the DEMO plasma. Techniques that still seem feasible without large modifications from the present practice are microwave techniques and direct line-of-sight techniques (e.g. neutrons, gamma and x-rays provided the detectors are not too close to the plasma).

Table 2. Overview of the main parameters of ITER and DEMO as compared to the best/highest achieved values in present devices (derived from ref. 10).

\begin{tabular}{|c|c|c|c|c|}
\hline Parameter & Parameter & ITER & $\begin{array}{l}\text { DEMO } \\
\text { Steady state }^{a}\end{array}$ & $\begin{array}{l}\text { Best achieved } \\
\text { individual } \\
\text { parameter }\end{array}$ \\
\hline Plasma volume & $\mathrm{V}\left(\mathrm{m}^{3}\right)$ & 850 & $900-2700$ & 80 (JET) \\
\hline Pulse length & (s) & $400-3000$ & c.w. & 390 (Tore Supra) \\
\hline Fusion power & $\mathrm{P}_{\text {fus }}(\mathrm{MW})$ & $\sim 500$ & $2500-5000$ & 16 (JET) \\
\hline Power multiplication & $\mathrm{Q}=\mathrm{P}_{\text {fus }} / \mathrm{P}_{\text {in }}$ & 10 & $15-35$ & 0.8 (JET) \\
\hline Total number of neutrons & $(\mathrm{n} / \mathrm{s})$ & $1.4 \times 10^{21}$ & $1.4-7 \times 10^{21}$ & $1.2 \times 10^{19}$ \\
\hline Neutron flux on first wall & $\left(\mathrm{n} / \mathrm{m}^{2} \mathrm{~s}\right)$ & $3 \times 10^{18}$ & $3-10 \times 10^{18}$ & $3 \times 10^{17}(\mathrm{JET})$ \\
\hline Neutron load on first wall & $\left(\mathrm{MW} / \mathrm{m}^{2}\right)$ & $\sim 0.5$ & $1-3$ & $\sim 0.05$ (max) $(\mathrm{JET})$ \\
\hline Neutron fluence & $\left(\right.$ MWyear $\left./ \mathrm{m}^{2}\right)$ & 0.3 & $5-15$ & negligible \\
\hline Neutron fluence & $\left(\mathrm{n} / \mathrm{m}^{2}\right)$ & $\sim 3 \times 10^{25}$ & $50-150 \times 10^{25}$ & $\sim 3 \times 10^{21}(\mathrm{JET})$ \\
\hline Displacements per atom in first wall & (dpa) & $\sim 3$ & $50-150$ & 0 \\
\hline
\end{tabular}

${ }^{\mathrm{a}}$ Since there is not yet a single steady state DEMO design, ranges of values are given here, covering the various options.

As it is mainly the neutron fluence (and not the flux) that is higher in DEMO, there may be prospects for use of in-vessel diagnostic components such as magnetic coils and bolometers if provisions can be made for them to be replaced regularly, or alternatively if they are only inserted and used intermittently. The same is true for plasma facing mirrors. If such in-vessel diagnostics and optical diagnostics with plasma facing mirrors are deemed essential for DEMO, schemes must be developed for regular replacement, which may significantly impact on the DEMO design. One could consider sparse, very constrained views, where conventional windows can survive, or be replaced during operation by having identical views at different locations. These systems could then be used and maintained cyclically, but there would be a significant reduction in measurement capability.

Another important effect is related to the high plasma temperatures in DEMO, leading to prominent relativistic effects, that limits the operational space of microwave diagnostics such as electron cyclotron emission and reflectometry; amongst others because of the strongly downshifted emission. Moreover, in present devices there is an apparent discrepancy between the electron temperature $\mathrm{T}_{\mathrm{e}}$ measured by ECE and by Thomson scattering, which sometimes occurs at high temperatures. ${ }^{11,12}$ This could be due to deviations from a Maxwellian velocity distribution driven by 
high levels of anisotropic additional heating power. Experiments on ITER at high temperatures and high levels of additional heating power should give insight into the cause of this discrepancy.

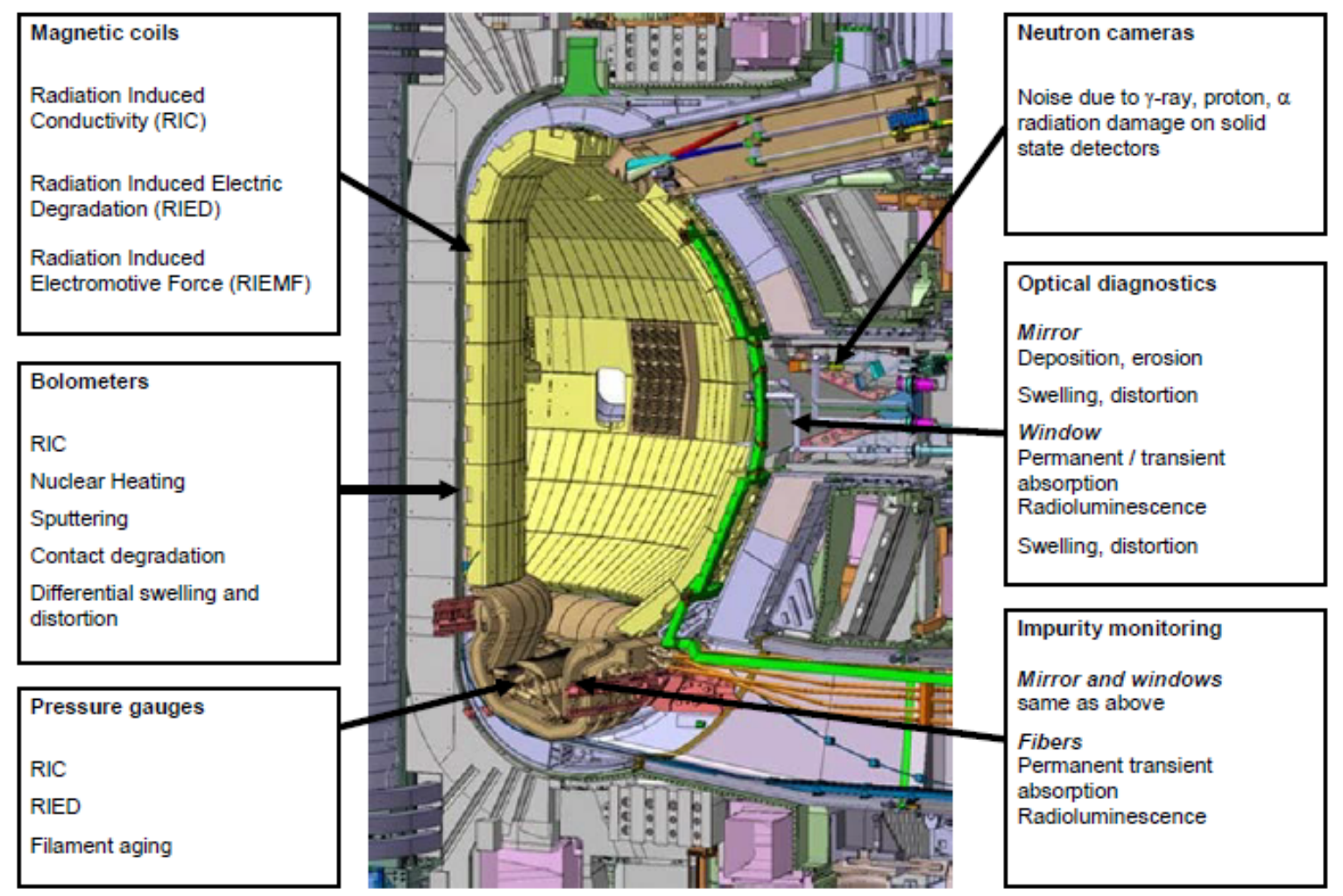

Figure 2. Main environmental effects on diagnostics and diagnostic components located at close proximity to the ITER plasma.

It may be evident that due to the multitude of environmental effects many conventional diagnostic techniques cannot be applied in DEMO (or only with severe restrictions). Therefore the measurement capability in DEMO may be less accurate and have less coverage than in present devices and ITER. Detailed profile, shape and equilibrium information may not be readily available to support complex plasma scenarios. Moreover, input for feedback control loops may be rather imprecise and this could have immediate consequences for the actuators used in control loops. But unlike ITER, DEMO will not be designed as a flexible research tool, since its main purpose is to demonstrate the technology of the fusion reactor. It is expected that DEMO will have only one or two different plasma operating scenarios. These scenarios will be developed and tested on the supporting research tokamaks and particularly on ITER. The limited number of operating scenarios implies that probably a smaller number of measurements and hence diagnostics will be required, since it will no longer be necessary to measure the full profile of as many different plasma parameters; instead it is possible that only those measurements that serve as indicator that DEMO is in the proper plasma operating scenario will be required.

Once the DEMO scenarios have been selected generically, one should try to define the minimum set of measurements needed to be able to operate DEMO in those scenarios, and thus assist the final scenario choices. In the first instance this could be done in a synthetic way by trying to study in a computer environment whether it is possible to still operate tokamaks and especially ITER in the DEMO scenarios by reducing the number of diagnostics and/or measurement channels/chords. One should bear in mind, that the lack of suitable diagnostic techniques may affect the options for 
controlling the DEMO plasma, in particular the ability to run "advanced" scenarios. This lack of feasible diagnostics, and thus the reduced set of plasma parameters that can be measured, will make modelling more important in DEMO to derive parameters that are needed but cannot be measured directly. Advanced predictive/analysis codes need to be developed to combine data from various diagnostics in an intelligent way in order to reduce the number of required diagnostics. Benchmarking of this should be done on ITER and elsewhere.

\section{Plasma effects that are special to DEMO}

Another effect that has an important influence on control of DEMO plasmas is that the power generated by the fusion reactions substantially exceeds the external input power (e.g. $\mathrm{P}_{\text {fusion }} / \mathrm{P}_{\text {in }} \sim 15$ 35). Twenty percent of the generated fusion power is carried by the charged alpha particles that carry a large fraction of the plasma kinetic energy and can collectively drive certain types of magnetohydrodynamic (MHD) modes, while they can suppress other MHD modes. In some cases these MHD modes can have both desirable effects on the plasma, as well as be detrimental to the plasma. For example, the so-called sawtooth instability on the one hand, if it occurs, could be largely responsible for the transport of the thermalized alpha particles (ash) and other impurities out of the core, but on the other hand may result in the loss of the energetic alphas before they have fully thermalized. Although several DEMO designs are presently designed to have a minimum q-value of 1.5 in the plasma, and hence are by definition sawtooth free, it is not unlikely that operation below $q=1$ is needed to have moderate sawteeth in order to remove the thermalized helium. An undesirable effect of the sawtooth instability is that it may trigger other MHD modes such as neoclassical tearing modes (NTMs). The NTMs in turn are detrimental to the plasma confinement and in some cases could lead to disruptive termination of the plasma. At the edge of the plasma, so-called edge localized modes (ELMs) can occur, which can result in extremely high transient heat and particle loads on the plasma facing components of a reactor, but can also assist with impurity control. Active control or avoidance of these modes is required to balance their desired and detrimental effects. The scientific challenge in the field of burn control is to find the proper balance between desired and detrimental effects of the various MHD modes and to develop methods and tools for active feedback control of such modes. An important complication arises from the fact that the external heating power is small compared to the heating power of the alpha particles and so the effectiveness of the external power as a control mechanism may be limited. To be able to develop control strategies, it is necessary to understand the dynamics of the system, in this case the mutual interactions between the fast alpha particles and the MHD instabilities.

\section{Development of diagnostics for DEMO}

In order to meet the requirements for measurements on DEMO, it is likely that new diagnostic techniques will need to be developed that are suitable for the DEMO environment, in particular to replace the established diagnostic techniques that will be problematic in DEMO. For in-vessel components, there may be little prospect other than ensuring they can be replaced frequently as was discussed in Sec. II. For ex-vessel components, the situation may be less critical. Nevertheless, this is an area that needs intensive study, requiring testing and qualification on fission reactors, and especially on ITER (e.g. during the full-power DT phase). Some testing of small components may also be possible in the International Fusion Materials Irradiation Facility (IFMIF) ${ }^{13}$. It should be noted that irradiation testing, even at ITER-relevant levels, is time consuming and costly. At the end of the 
DEMO Conceptual Design Activity one has to have a good idea of which diagnostics will work and how they can be integrated into DEMO; therefore the irradiation testing is not only important, but may also be urgent. Work on the supporting research tokamaks and ITER needs to guide the selection of techniques that can best cope with the harsh environment. During the ITER lifetime, experience will be gained with real-time data handling and validation to process large quantities of data, in-pulse calibration, etc. It is likely that some DEMO-relevant diagnostics will not provide measurements with the same quality as non-DEMO-relevant diagnostics on present devices, and therefore will not be of benefit as a diagnostic on those devices. Nevertheless, such new diagnostic techniques must be developed, and this aspect should become an accepted part of the fusion programme.

To circumvent the problems encountered in optical systems with either refractive components (vulnerable to RIA and/or RIE) or mirrors (vulnerable to erosion, deposition and swelling), one could consider using freestanding metallic transmission gratings, which can be produced by electron beam lithography and have up to $10^{4}$ lines/mm. ${ }^{14,15}$ Other possibilities that could be explored are metal Fresnel zone plates, ${ }^{16}$ or so-called photonic sieve metallic lenses. ${ }^{17}$ The drawback of these optical components is that their optical transmissivity is less than that of ordinary lenses and mirrors. However, they might be robust enough to withstand the exposure to the burning plasma with possibly less degradation with time of their light collecting and polarizing properties than conventional optics. An option for the transport of visible light through complicated trajectories could be the application of hollow optical fibres. ${ }^{18}$ Photonic-crystal hollow fibres ${ }^{19,20}$ can nowadays be produced thanks to advances in nanotechnology. In these fibres the light wave is guided by constructive interference on a layer of sub-wavelength channels surrounding a larger hollow core. It is claimed that up to about $98 \%$ of the light energy is guided through the hollow core, due to a photonic band gap effect. ${ }^{19,20}$ Of course these newly proposed solutions need to undergo irradiation testing to judge whether indeed they are feasible in a high radiation environment.

Some of the DEMO designs assume that there will not be neutral heating beams and that all additional heating will be supplied by radiofrequency techniques. If the type of data obtained in ITER and present day machines with diagnostics that use neutral beams (for example $T_{i}, v_{\text {rot }}, B_{p}$, He-content) are deemed important for DEMO, techniques and hardware will need to be developed to obtain the same information without neutral beams. For instance, one could pose the question whether it is possible to use measurement of He near the edge to control the He ash in the plasma core?

It is expected that several of the measurements in DEMO will be rather fuzzy, ${ }^{21,22}$ i.e. not precise, and that sharpening based on forward modelling of various types is needed, ${ }^{23,24}$ or alternatively by introducing automated consistency checks between multiple independent fuzzy data types. Also rather unconventional approaches have been proposed including the use of calorimetry maps for determining the neutron source function. ${ }^{25,26,27}$ Due to the substantial contribution from thermal radiation from the plasma which affects the calorimetry on a slower timescale than the neutrons, however, calorimetry cannot be categorized as a neutron diagnostic. Nevertheless, it can directly and accurately assess the global energy balance of a discharge and it can be also used to measure the heat decay time and therefore still could be considered as a rather easy to implement diagnostic. $^{26,27,28}$

The number of diagnostics ports in DEMO will be rather limited and so the available space needs to be used efficiently. An assessment of the minimum required space for diagnostics for a DEMO reactor based on the advanced tokamak scenario is presented in ref. ${ }^{29}$. A good example of the efficient use of a port is the dual use of a port for heating and diagnostics. The Electron Cyclotron Emission (ECE) from the plasma is measured via the injection line of Electron Cyclotron Resonance 
Heating (ECRH) (Fig. 3). ${ }^{30,31}$ The system enables the response of the plasma due to localised ECRH (e.g. the suppression of neoclassical tearing modes or the control of sawteeth) to be measured without the need to map the measurement onto the heating location via the magnetic equilibrium. The system has been successfully applied in TEXTOR to measure the ECE radiation at the $\mathrm{nW}$ level via the injection line for $1 \mathrm{MW}$ of ECRH power. ${ }^{30,31}$ Although the direct measurement of the plasma response at the point of ECRH deposition was the main motivation to build this system, an additional benefit is that a single access port is used by two systems. A collinear ECE/ECRH system for utilization in a waveguide environment with CW heating is presently in preparation for implementation on ASDEXUpgrade. $^{32}$ This system is meant to be also compatible for use in ITER and possibly also in DEMO.

There are also possibilities to control plasma phenomena without the use of elaborate diagnostic systems. A good example is the sawtooth pacing study that has been recently performed at the TCV tokamak by means of open loop control. ${ }^{33}$ In this study the sawtooth period could be controlled within a certain bandwidth using modulated ECRH with the power deposited just outside the $q=1$ radius. This technique is known as "pacing". For certain combinations of ECRH power, duty cycle and modulation period, the sawtooth response to the ECRH modulation was essentially instantaneous. Only a very simple diagnostic, such as a few chord integrated x-ray measurements, is needed to be able to monitor the sawtooth period and radius.

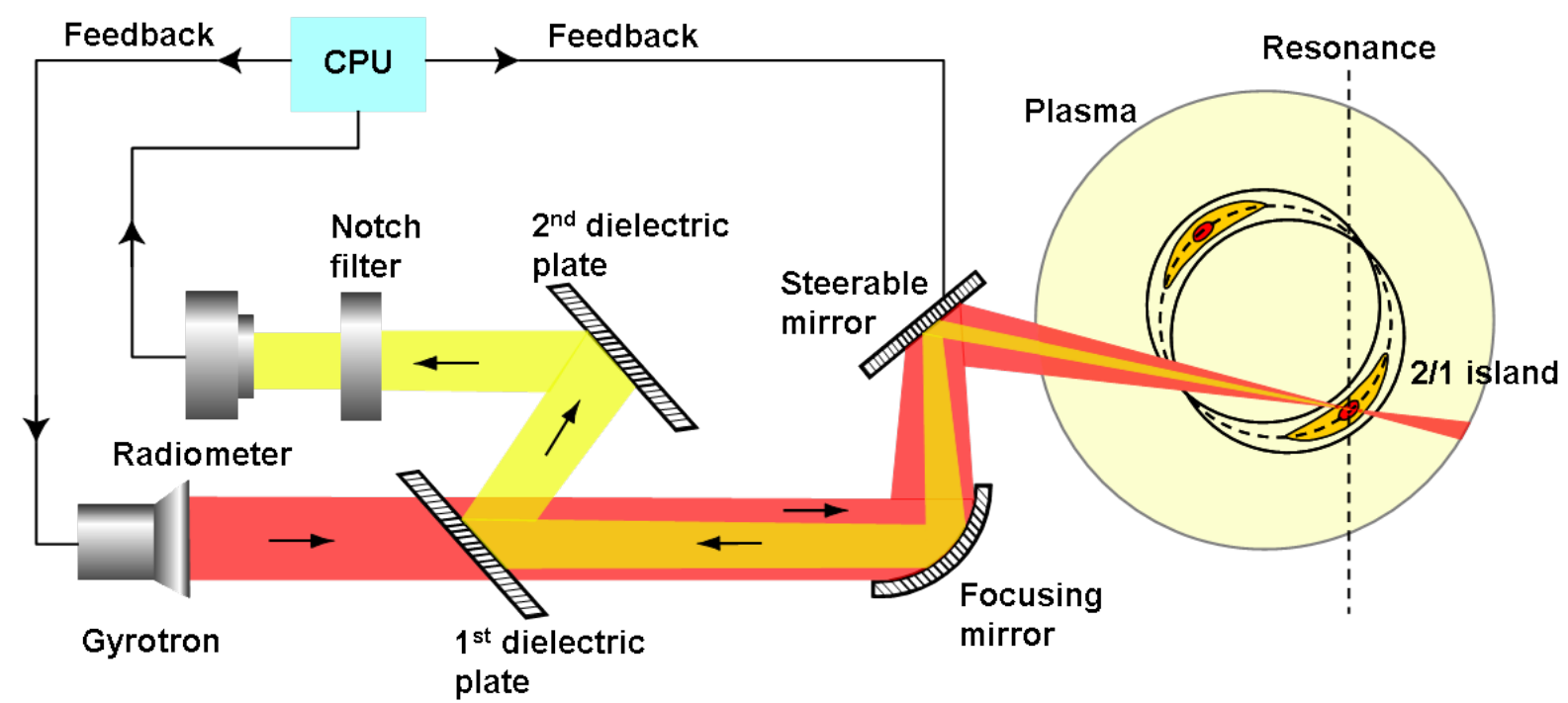

Figure 3. Collinear ECE/ECRH system for tearing mode suppression at TEXTOR. ${ }^{30,31}$ The yellow path carries nanoWatt ECE signals, the red one Megawatt ECRH radiation.

In the first years of DEMO operation, during the low activation phase, many conventional diagnostics might still work and these systems could be used to develop and exploit the DEMO scenarios. At the same time these systems can be used to train new radiation hard techniques or gain experience with using combinations of fuzzy measurements to determine some plasma parameters. Some of the conventional diagnostics will become progressively unusable during the transition from low to high activation phase, but they could continue to be used as long as they are functioning. For some conventional systems, it is possible to consider intermittent measurements (e.g. "dipstick" method) rather than having continuous data. 


\section{Design Process}

An overview of the design and R\&D process that will be required for diagnostics is shown in Figure 4, which has been adapted from ref. 8. (A slightly different, but in gross lines rather similar, version of this process is being applied in a study of diagnostics for a Fusion Power Plant and is described in ref. 5). The left part of the flow diagram is basically the process that is presently being used for ITER. It is assumed here that all diagnostics on DEMO are used for control purposes and that no diagnostics are implemented purely for physics evaluation, as is still the case on ITER.

The requirements flow from the functional role anticipated for the diagnostic measurements in the operation of the machine; for example, measurements for device protection and plasma control purposes. As part of the initial conceptual design of the machine, a well justified set of measurements requirements is developed. Selection of diagnostic systems to meet the requirements follows. In this process the environment is taken into account and diagnostic systems are chosen that offer the best possibility of operation, or require minimum $R \& D$ to achieve operational status, in the harsh environment. Conceptual design of the individual systems and integration into the machine design follows. In the integration, diagnostic components are designed for installation at various locations for example in-vessel, in diagnostic ports and in the divertor - and in these locations will unavoidably be subject to different levels of thermal, mechanical and radiation loads. The performance of the integrated design and especially the ability of the individual diagnostic systems to operate in the harsh environment are assessed. If the integrated design is assessed to be able to meet requirements detailed design can follow. If, however, there is a significant shortfall of performance relative to requirements in specific areas, then the associated requirements are reassessed carefully to ensure that they have not been set too stringently. If they have, then perhaps they can be relaxed and loop A closed by diagnostic reselection and design. If loop A cannot be closed then it is necessary to return to the initial step and adjust the machine and/or plasma design at a higher level (loop B) to improve the diagnostic implementation or adapt the control requirements. The goal is to achieve a set of measurements requirements that meets the expected operational needs for the machine and a diagnostic set that can be implemented with minimum R\&D.

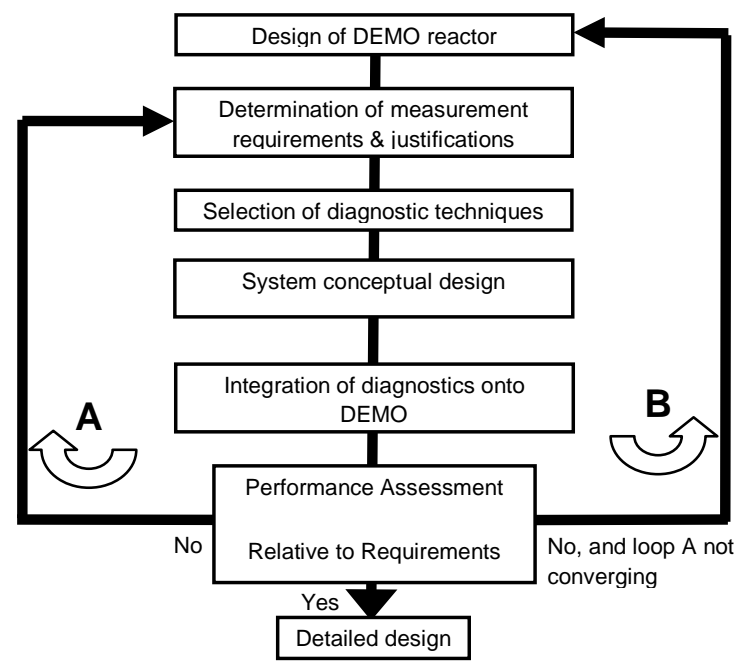

Figure 4. Flow diagram for the design and R\&D of control diagnostics on DEMO (adapted from ref. 8)

It is important to develop a new way of thinking with respect to that employed to date in which diagnostics are added after the machine and plasma scenario have been basically designed. 
Even in ITER the diagnostics set has been designed mainly using Loop A in Figure 4 (albeit that for some diagnostics small modifications to some ports, blanket modules or the divertor have been necessary). The harsher environment in DEMO, combined with the expected higher level of real-time control, means that the needs and implementation difficulties in diagnostics have to be taken into account during the entire design phase: for example, their replaceability or the required screening against radiation may have a large impact on the DEMO design. In other words, Loop B should be explicitly included in the design of the machine combined with its set of diagnostics.

\section{Conclusions}

Due to the relatively harsh environment especially in the vacuum vessel, combined with the requirements for high availability and low maintainability, the design and implementation of control diagnostics on a DEMO will be a major challenge. Some established diagnostic techniques that are used extensively in current research to provide basic plasma measurements may not be applicable and new techniques will be required. In any case, extensive design and R\&D focused on the specific problems of implementing diagnostics in the harsh environment will be needed. Many diagnostics will be part of critical control loops in DEMO. Hence, given the generic difficulty in their implementation due to the multitude of environmental effects, it is an absolute necessity to include diagnostics into the DEMO project and the plasma scenario design from the very beginning of the conceptual design phase. In DEMO it is no longer possible to regard diagnostics as an afterthought, since this potentially could lead to a machine that cannot be operated since its parameters cannot be adequately controlled.

\section{Acknowledgement}

This work, supported by the European Communities under the contracts of Association between EURATOM and FOM and between EURATOM and CCFE, was carried out within the framework of the European Fusion Program and was funded partly by NWO-RFBR grant 047.018 .002 and by the RCUK Energy Programme under grant EP/I501045. The views and opinions expressed herein do not necessarily reflect those of the European Commission 


\section{References}

1 D. Maisonnier et al., Nucl. Fusion 47 (2007) 1524 - 1532.

2 F. Najmabadi et al., Fusion Eng. Design 80 (2006) 3 - 23.

$3 \quad$ K.M. Feng et al., Fusion Eng. Design 84 (2009) 2109 - 2113.

$4 \quad$ K. Tobita et al., Fusion Eng. Design 81 (2006) 1151 - 1158.

5 T. Brown et al., submitted for publication in IEE Transact. on Plasma Sci. (2011).

$6 \quad$ A. Sagara et al., Fusion Eng. Design 83 (2008) 1690 - 1695.

G. Federici et al., private communication (part of the EFDA Work Programme 2012)

A.J.H. Donné et al., Nucl. Fusion 47 (2007) S337 - S384.

G. Vayakis et al.,Fus. Sci. Techn. 53 (2008) $699-750$.

38 (2010) $2934-2943$

E. de la Luna et al., Proc. $15^{\text {th }}$ Joint Workshop on Electron Cyclotron Emission and Electron Cyclotron Resonance Heating (2008), Yosemite, USA, (World Scientific Publishing, Singapore, 2009), pp 200 - 207.

G. Taylor et al., Plasma Phys. Control. Fusion 36 (1994) 523 - 542.

A. Moeslang et al., Fusion Eng. Des. 81 (2006) 863 - 871.

D. Stutman et al., Rev. Sci. Instrum. 76 (2005) 023505.

T. Wilhein et al., Rev. Sci. Instrum. 70 (1999) 1694.

L. Kipp et al., Nature 414 (2001) 184 - 188.

K. Kincade, Laser Focus World 40 (2004) 34.

A. Ramsey, Rev. Sci. Instrum. 66 (1995) $871-876$.

C. M. Smith et al., Nature 424 (2003) 657 - 659.

J.D. Joannopoulos et al., Nature 386 (1997) 143 - 149.

R. Viertl, Statistical methods for fuzzy data, Wiley, West Sussex, UK, ISBN 978-0-47069945-4.

Publishers, ISBN 978-1-60741-900-6.

F. Russo, IEEE Transact. On Instrumentation and Measurement 51 (2002) 824 - 828.

Z. Gui and J. Liu, Optik - Int. J. for Light and Electron Optics 122 (2011) 697 - 702.

J.M. Richardson et al., Proc. IEEE Nuclear Sci. Symp. (1997) Albuquerque, USA, Vol. 1, pp $135-139$.

27 J.C. Vallet, private communication (presented at $8^{\text {th }}$ ITPA Diagnostics Topical Group Meeting, Culham, UK, 2005)

M. Sasao, private communication (presented at the $9^{\text {th }}$ ITPA Diagnostics Topical Group Meeting, Daejon, Korea, 2005) (2010) Rome, Italy, paper FR-E1.1.

M. Lauret et al., submitted for publication in Nucl. Fusion (2012). 